\title{
SYMBOLIC COMPUTATION
}

Artificial Intelligence

Managing Editor: D. W. Loveland

Editors: S. Amarel

A. Biermann L. Bolc

A. Bundy

H. Gallaire

P. Hayes

A. Joshi

D. Lenat

A. Mackworth

R. Reiter

E. Sandewall

J. Siekmann

W. Wahlster 
R.S. Michalski, J.G. Carbonell, T.M. Mitchell (Eds.): Machine Learning. An Artificial Intelligence Approach. XI, 572 pages, 1984

A. Bundy (Ed.): Catalogue of Artificial Intelligence Tools. Second, revised edition. IV, 168 pages, 1986

C. Blume, W. Jakob: Programming Languages for Industrial Robots. XIII, 376 pages, 145 figs., 1986

J.W. Lloyd: Foundations of Logic Programming. Second, extended edition. XII, 212 pages, 1987

L. Bolc (Ed.): Computational Models of Learning. IX, 208 pages, 34 figs., 1987

L. Bolc (Ed.): Natural Language Parsing Systems. XVIII, 367 pages, 151 figs., 1987

N. Cercone, G. McCalla (Eds.): The Knowledge Frontier. Essays in the Representation of Knowledge. XXXV, 512 pages, 93 figs., 1987

G. Rayna: REDUCE. Software for Algebraic Computation. IX, 329 pages, 1987

D.D. McDonald, L. Bolc (Eds.): Natural Language Generation Systems. XI, 389 pages, 84 figs., 1988

L. Bolc, M.J. Coombs (Eds.): Expert System Applications. IX, 471 pages, 84 figs., 1988

C.-H. Tzeng: A Theory of Heuristic Information in Game-Tree Search. X, 107 pages, 22 figs., 1988

L. Kanal, V. Kumar (Eds.): Search in Artificial Intelligence. X, 482 pages, 67 figs., 1988

H. Coelho, J. Cotta: Prolog by Example. 304 pages, 62 figs., 1988

H. Abramson, V. Dahl: Logic Grammars. XV, 258 pages, 40 figs., 1989

R. Hausser: Computation of Language. 425 pages, 1989

B. D'Ambrosio: Qualitative Process Theory Using Linguistic Variables. 156 pages, 22 figs., 1989 


\section{Bruce D'Ambrosio}

\section{Qualitative Process Theory Using Linguistic Variables}

With 22 Figures

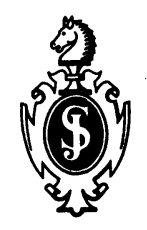

Springer-Verlag

New York Berlin Heidelberg London Paris Tokyo Hong Kong 


\author{
Bruce D'Ambrosio \\ Department of Computer Science \\ Oregon State University \\ Corvallis, OR 97331-4602
}

Library of Congress Cataloging-in-Publication Data

D'Ambrosio, Bruce.

Qualitative process theory using linguistic variables.

(Symbolic computation. Artificial intelligence)

Includes bibliographical references.

1. Artificial intelligence. 2. Reasoning.

3. Linguistics. I. Title. II. Series.
Q335.D36 1989
006.3
89-21698

Printed on acid-free paper

(C) 1989 Springer-Verlag New York Inc.

Softcover reprint of the hardcover 1st edition 1989

All rights reserved. This work may not be translated or copied in whole or in part without the written permission of the publisher (Springer-Verlag New York, Inc., 175 Fifth Avenue, New York, NY 10010, USA), except for brief excerpts in connection with reviews or scholarly analysis. Use in connection with any form of information storage and retrieval, electronic adaptation, computer software, or by similar or dissimilar methodology now known or hereafter developed is forbidden. The use of general descriptive names, trade names, trademarks, etc., in this publication, even if the former are not especially identified, is not to be taken as a sign that such names, as understood by the Trade Marks and Merchandise Marks Act, may accordingly be used freely by anyone.

Camera-ready text prepared by the author using $T_{E} X$.

$\begin{array}{lllllllll}9 & 8 & 7 & 6 & 5 & 4 & 3 & 2 & 1\end{array}$

ISBN-13: 978-1-4613-9673-4

e-ISBN-13: 978-1-4613-9671-0

DOI: 10.1007/978-1-4613-9671-0 


\section{Contents}

1 Overview 1

2 Fuzzy Logic Control 5

2.1 Classical Control Theory . . . . . . . . . . . . . . . 5

2.2 A New Approach to Control of Complex Systems . . . . . . 6

2.3 Fuzzy Control . . . . . . . . . . . . . . . . . 7

2.3.1 Fuzzification of Input . . . . . . . . . . . . 9

2.3.2 Rule Interpretation . . . . . . . . . . . . . . 9

2.3.3 Defuzzification ... . . . . . . . . . . . . 10

2.4 Extensions of the Fuzzy Control Paradigm . . . . . . . . . 12

2.5 The Next Step: Fuzzy-Model-Based Control . . . . . . . . . 13

3 Introduction to Qualitative Process Theory 16

3.1 Uses of QP Theory . . . . . . . . . . . . . . . . 18

3.2 Process versus Device-Centered Theories . . . . . . . . . . 19

3.3 Qualitative Process Theory - Definitions and Examples . . . 21

3.4 Reasoning in QP Theory . . . . . . . . . . . . . 25

3.5 Historical Background . . . . . . . . . . . . . 28

3.6 Summary .................... 30

4 Application of QP Theory to Process Control - An Example $\quad 31$

4.1 The Reaction Process . . . . . . . . . . . . . 34

4.2 Countercurrent Heat Flow . . . . . . . . . . . . . . 35

4.3 Basic Deductions . . . . . . . . . . . . . . 35

5 Ambiguity in QP Theory 44

5.1 Ambiguity in QP Theory . . . . . . . . . . . 44

5.2 Representation Alternatives . . . . . . . . . . . . 46

5.2.1 Representations for Uncertainty . . . . . . . . . . . 46

5.2.2 Representations for Parameters of Physical Systems 48

5.2.3 Characterizations of Functional Relationships . . . . 50

5.3 Related Work . . . . . . . . . . . . . . . . . . . 52 
5.4 Linguistic Extensions to QP Descriptions . . . . . . . 53

6 Linguistic Variables 56

6.1 Introduction to Linguistic Variables . . . . . . . . . 56

6.2 Approximate Reasoning and the Compositional Rule of In-

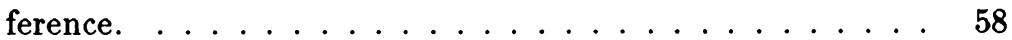

6.3 Similarity of Linguistic Variables and Relations . . . . . . 60

6.4 Support-Pair Certainty . . . . . . . . . . . . . . . 61

6.4.1 Support Logic Programming . . . . . . . . . . 63

6.4.2 Support-Pair Certainty and Fuzzy Sets . . . . . . . . 64

6.4.3 Relationship of SLP with Linguistic Variables and the Compositional Rule of Inference . . . . . . . 66

6.5 Truth Maintenance with Numeric Certainty Estimates . . . 66

6.5.1 Review of Methods for Uncertainty Management in AI 69

6.5.2 Assumption-Based Truth Maintenance . . . . . . 69

6.5.3 SLP and Assumption-Based Truth Maintenance . . 73

6.5.4 Symbolic Computation of Belief Functions . . . . . . 75

6.5 .5 Example . . . . . . . . . . . . . . 78

6.5.6 Discussion and Related Research _. . . . . . . . 78

6.6 Summary . . . . . . . . . . . . . . . 81

$\begin{array}{lll}7 & \text { Linguistic Quantity Spaces } & 82\end{array}$

7.1 A Functional Overview . . . . . . . . . . . . . . . 82

7.2 Computing the Consequences of a Linguistic Quantity Space 83

7.2.1 Representation of Parameter Values and Relations . 83

7.2.2 Quantity Space Deductions . . . . . . . . . . . 84

7.2.3 Implementing the Quantity Space Deductions . . . . 90

7.3 Linguistic Measurement Interpretation . . . . . . . . . 91

8 Characterization of Functional Relationships 96

8.1 Sources of Ambiguity in QP Theory Models . . . . . . . . 96

8.1.1 Ambiguity 1 - Offgas Exit Temperature . . . . . . 97

8.1.2 Ambiguity 2 - Product Temperature . . . . . . . . 100

8.1.3 Ambiguity 3 - Material-Flow Rates . . . . . . . . . 102

8.1.4 Discussion . . . . . . . . . . . . . . . . . . 104

8.2 Fuzzy Relational Algorithms . . . . . . . . . . . 105

8.2.1 Computing Individual Influence Effects . . . . . . 107

8.2.2 Adjusting for Description Applicability . . . . . . 108

8.2.3 Combining Influence Effects . . . . . . . . . . . 109

8.3 Annotation Management . . . . . . . . . . . . . 110

8.4 Examples . . . . . . . . . . . . . . . . 113

8.5 Summary . . . . . . . . . . . . . . 118 
9 Qualitative Perturbation Analysis 120

9.1 Qualitative Perturbation Analysis . . . . . . . . . . . 120

9.2 Extended Perturbation Analysis . . . . . . . . . . 123

9.3 Linguistic Perturbation Analysis . . . . . . . . . . . 131

9.4 LPA Extended Example . . . . . . . . . . . . . . . . 134

9.4 .1 Input Script . . . . . . . . . . . . . . . . . 134

9.4 .2 Output Trace . . . . . . . . . . . . . 137

9.5 Summary . . . . . . . . . . . . . . . . . 141

10 Evaluation and Conclusion 142

10.1 Review . . . . . . . . . . . . . . . . . . 142

10.2 Evaluation . . . . . . . . . . . . . . 143

10.3 Further Research . . . . . . . . . . . . . . . . . 145

10.4 Conclusion . . . . . . . . . . . . . 146

10.5 References . . . . . . . . . . . . . . . . 147 


\section{List of Figures}

1.1 Boiling Example, after Forbus [For84] . . . . . . . . . 2

2.1 Classical Model of Process Control . . . . . . . . . . . 5

2.2 A Model of Fuzzy Logic Control . . . . . . . . . . . 8

2.3 Possible Membership Functions for Fuzzy Control Actions . 11

2.4 Fuzzy-Model-Based Control . . . . . . . . . . . . . 14

3.1 Contained-Liquid View . . . . . . . . . . . . . . 22

3.2 Heat-Flow Process . . . . . . . . . . . . . . . . 25

4.1 A Simplified Chemical Furnace . . . . . . . . . . . 32

4.2 Chemical Furnace Initial Situation Description . . . . . . . 33

4.3 The Reaction Process . . . . . . . . . . . . . 36

4.4 Countercurrent Heat Flow . . . . . . . . . . . . . . . 37

4.5 Definition of Contained Substances . . . . . . . . . . . 38

4.6 Influence Dependency Graph . . . . . . . . . . . 41

4.7 Results of QP Analysis of Furnace . . . . . . . . . . 42

5.1 Arithmetic for Qualitative Sign Abstractions . . . . . . 52

6.1 Semantic Rule for a Linguistic Variable . . . . . . . . . 57

6.2 Syntactic Rule as a Production System . . . . . . . . . . 58

6.3 Containment . . . . . . . . . . . . . . . 62

6.4 Combining Independent Bodies of Evidence . . . . . . . 65

6.5 Support-Pair Containment Tests . . . . . . . . . . . 67

6.6 Composition Rule of Inference as a Set of SLP Implications 68

6.7 Simple Label Propagation in an ATMS . . . . . . . . . . . 71

6.8 Representing Mutual Exclusion in an ATMS . . . . . . . . 72

6.9 Assumptions for a Basic Mass Assignment . . . . . . . . . . 74

6.10 Representing an SLP Inference Rule in the ATMS . . . . 76

6.11 Symbolic Computation of Belief Functions . . . . . . . . 77

6.12 Example Problem Setup . . . . . . . . . . . . . . 79

6.13 ATMS Example Results . . . . . . . . . . . . . . 80

7.1 Defining an Ordering Relation Inference Matrix . . . . . . 84 
7.2 Definition of strictlylessthan over $\left\{\begin{array}{lllll}10 & 20 & 30 & 40 & 50\end{array}\right\} \ldots \ldots$

7.3 Sample Quantity Space . . . . . . . . . . . . . . 86

7.4 Construction of Quantity Spaces . . . . . . . . . . . 93

7.5 Interpretation of Linguistic Quantity Ordering Information 94

7.6 State Details for Linguistic Measurement Interpretation . . 95

8.1 Ambiguity Source 1 - The Conflict Triangle . . . . . . . 98

8.2 The Conflict Triangle Annotated with Influence Sensitivities 100

8.3 Ambiguity 2 - Loop Pair Case 1 . . . . . . . . . . . . . 101

8.4 Loop Pair Type 1 - With Annotations . . . . . . . . . 103

8.5 Ambiguity 3 - Loop Pair Type 2 . . . . . . . . . . . . 104

8.6 Loop Pair Type 2 - With Annotations . . . . . . . . . 105

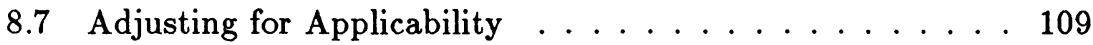

8.8 Combining Influences . . . . . . . . . . . . . . . . 111

8.9 Combining influences graphically . . . . . . . . . . . 112

8.10 Extended Influence Resolution Algorithm . . . . . . . . . 114

8.11 Influence Resolution without Annotations . . . . . . . . 115

8.12 Annotating the Influences . . . . . . . . . . . . 116

8.13 Influence Resolution with Annotations . . . . . . . . . . 117

8.14 Comparison of Results of Influence Resolution . . . . . . . 119

9.1 Simple Qualitative Perturbation Analysis Algorithm . . . . 122

9.2 QP Sample Influence Graph . . . . . . . . . . . . . . 123

9.3 Revised Qualitative Perturbation Analysis Algorithm . . . . 124

9.4 Annotation of EPA for Heater Temp Increase . . . . . . . . 126

9.5 Results of EPA on Heater Temp Increase . . . . . . . . 127

9.6 Annotation for Reactant Temp Increase . . . . . . . . . . 128

9.7 Results of Extended QPA on Reactant Temp Increase . . 129

9.8 Extended QPA Example Summary . . . . . . . . . . 130

9.9 Linguistic Perturbation Analysis Example . . . . . . . . 135 